\title{
Strategies to keep kidney transplant alive amid the SARS-CoV-2 pandemic
}

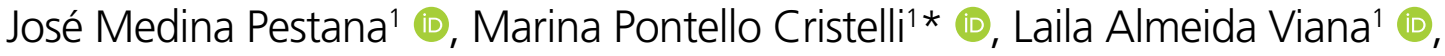

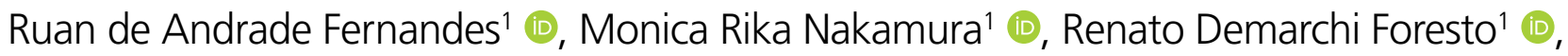 \\ Suelen Bianca Stopa Martins ${ }^{1}$ (1), Daniel Wagner de Castro Lima Santos ${ }^{1}[\mathbb{0}$, \\ Wilson Ferreira Aguiar ${ }^{1}$ (D), Maria Lucia dos Santos Vaz $^{1}$
}

\section{SUMMARY}

OBJECTIVE: This study aims to describe the result of the strategies adopted to maintain the transplant program amid the COVID-19 pandemic. METHODS: Since March 2020, several measures have been adopted sequentially, including the compulsory use of personal protective equipment and the real-time polymerase chain reaction testing of collaborators, symptomatic patients, potential deceased donors, candidates for recipients, and in-hospital readmissions, regardless of symptoms. The living-donor transplantation was restricted to exceptional cases. RESULTS: Among 1013 health professionals, 201 cases of COVID-19 were confirmed between March and August 2020, with no severe cases reported. In this period, we observed a $19 \%$ institutional increase in the number of transplants from deceased donors compared with that observed in the same period in 2019. There was no donor-derived severe acute respiratory syndrome virus (SARS-CoV-2) infection. Four COVID-19-positive patients underwent transplantation; after 28 days, all were alive and with functioning allograft. Among the 11,875 already transplanted patients being followed up, there were 546 individuals with confirmed diagnosis, 372 who required hospitalization, and 167 on mechanical ventilation, resulting in a $27 \%$ mortality rate.

CONCLUSIONS: These data confirm that the adoption of sequential and coordinated measures amid the pandemic was able to successfully maintain the transplant program and ensure the safety of health professionals and transplanted patients who were already in follow-up. KEYWORDS: Delivery of health care. Chronic kidney disease. Kidney transplantation. Coronavirus infections.

\section{INTRODUCTION}

On March 11, 2020, about 3 months after the first confirmed cases in Hubei Province, China, coronavirus disease 2019 (COVID19), a disease caused by severe acute respiratory syndrome virus (SARS-CoV-2), was declared a pandemic by the World Health Organization $^{1}$. In Brazil, the first confirmed case was registered on February 26 in São Paulo. On March 16, social distancing measures were adopted, with schools and offices closed, followed by bars and restaurants. The first death occurred on March 17 .

Currently, Brazil is the second country with the highest number of COVID-19 cases in the world, with infection widespread in all 27 states of the Union ${ }^{1}$. In addition to the direct effects of COVID-19, which include the occupation of infirmary beds and intensive care units by acute respiratory syndrome patients and the high consumption of personal protective equipment, it was possible to anticipate the magnitude of the impact of the pandemic on access to health services. This was especially true for services dedicated to the treatment of chronic and complex diseases, such as chronic kidney disease and kidney transplants.

According to the data from the Brazilian Dialysis Census, there are currently 133,464 patients on chronic dialysis treatment, with an average annual increase of 5587 patients in the

\footnotetext{
'Universidade Federal de São Paulo, Hospital do Rim - São Paulo (SP), Brazil.

*Corresponding author: ninacristelli@yahoo.com.br

Conflicts of interest: the authors declare there are no conflicts of interest. Funding: none.

Received on September 17, 2020. Accepted on September 20, 2020.
} 
last 10 years ${ }^{2}$. With the largest number of procedures, Brazil has the largest public kidney transplant system in the world, next only to United States. In the last 10 years, an annual average of 5703 kidney transplants were performed in the country ${ }^{3}$.

Hospital do Rim is a tertiary hospital located in the city of Sao Paulo. Since its foundation in 1998, more than 15,000 kidney transplants have been performed, with an average of 70 procedures a month and $70 \%$ of these kidney transplants involve a deceased donor ${ }^{4}$. However, there are still more than 9000 patients on the waiting list in this institution, and, in Brazil, approximately 30,000 candidates are waiting for the transplantation procedure ${ }^{2}$.

The direct and immediate risks of COVID-19 to the organ donation and transplantation program are numerous. From the risk of infection of the health professionals involved in the organ procurement and transplantation, as well as the risks associated with inadvertent transmission by the donor organ, the more intense immunosuppressive therapy received by the newly transplanted can increase the risk for infection in the context of chronic use of immunosuppressive drugs. Indirect and medium and long-term losses include the collapse of intrahospital organ search committees and transplant teams, the reduction in the number of potential donors, and the accumulation of dialysis patients in a scenario of lack of equipment and supplies.

\section{METHODS}

Aiming at maintaining the activity of organ procurement, donation, and transplantation and minimizing the risks to the transplantation program, the first and most decisive actions were taken on January 13, 2020, with the institution of a COVID-19 managing committee and daily pedagogical actions among employees and patients on concepts, forms of contagion, and institutional and community care in relation to the pandemic. The measures for higher impact in containing the spread of COVID-19 were taken by the institution on March 16,2020 , starting with the investigation of SARS-CoV-2 infection using real-time polymerase chain reaction (RT-PCR) tests for all employees and symptomatic transplanted patients, with test results being obtained within a period of $24 \mathrm{~h}$. In parallel, personal protective equipment, including N95 masks and faceshield masks, were made available for compulsory use by all professionals and patients within the institution. The investigation of respiratory symptoms and measurement of temperature and oximetry for all people was also instituted at the hospital.

\section{RESULTS}

There were 201 confirmed cases of COVID-19 among the 1013 employees of the institution, but only 125 among the 752 full-time permanent staff. In 56 of the 201 cases, the probable source of contagion was intrahospital interaction, demonstrating the efficiency of the measures adopted. Since this population presents low risk for complications, with an average age of 37 years, having overweight as the only comorbidity (99 employees, 49\%), the predominant symptomatology was mild; only 6 required hospitalization in a ward bed. All these 201 employees returned to work without sequelae.

Elective interventional transplant procedures were postponed from March 28, 2020 and performed only in exceptional situations, when the risk of infection of the recipient on dialysis was considered high due to their frequent displacement in the community. Thus, the number of transplants with live kidney donors was reduced from a monthly average of 28 to 4 procedures (Figure 1).

Inadvertent transmission of SARS-CoV-2 infection from the deceased donor to either a health care professional or to organ recipients was a major concern, as potential candidates remain in emergency or intensive care units days before the diagnosis of brain death as well as during the family authorization process for organ donation and extraction. Thus, since April 6, 2020, screening with RT-PCR using nasal swab of all potential donors prior to organ harvesting was instituted, with timely test results for donor exclusion in cases of confirmed infection. Since the beginning of screening until August 28, 2020, 269 deceased donors were tested, and 20 (7.5\%) were rejected after confirmation of SARS-CoV-2 infection. There has been no confirmed donor-derived SARS-CoV-2 infection in any transplant recipient.

A similar screening strategy has been applied to potential transplant recipients since April 20, 2020, with nasal swab collection for investigation of SARS-CoV-2 infection just before the surgical procedure. Initially, due to logistic limitations of obtaining the result in time to perform the surgery, the transplantation was performed in asymptomatic recipients screened by normal thoracic tomography, with the result of the RT-PCR test available after the transplantation. Between April 6 and June 12, 2020, 154 transplantations were performed without the result of the RT-PCR SARS-CoV-2 pretransplant test, and 3 of them were confirmed positive. After this period, the results of the RT-PCR were obtained before the surgery, except when the cold ischemia time was prolonged enough to compromise the graft viability. In this condition, another patient was submitted to transplantation without the result of the test due to a critical cold ischemia time of $36 \mathrm{~h}$ and was confirmed positive. Between April 6 and August 28, 2020, 20 potential recipients tested positive, with 16 being discarded and 4 of them undergoing transplantation in the conditions described earlier. In 28 days, these four patients were asymptomatic, with functioning renal graft and with the usual immunosuppression regime. 
A major issue concerns the decision to reduce the initial immunosuppression intensity of the transplant. The institutional decision was to maintain the protocols, composed of induction with $1 \mathrm{~g}$ of methylprednisolone intraoperatively, a single dose of $3 \mathrm{mg} / \mathrm{kg}$ of thymoglobulin in the immediate postoperative period, and maintenance with tacrolimus, prednisone, and azathioprine or mycophenolate. The rationale for this decision was the possibility of high risk of acute rejection of the graft associated with an eventual reduction of the immunosuppression intensity. During this period, patients were back to the community and were subjected to with high mobility for follow-up visits and thus were more exposed to the risk of acquiring viral infections. The adoption of this conduct in this 6-month period did not lead to a negative repercussion on the evolution of transplantations performed.

In the follow-up after transplantation, face-to-face elective consultations were limited to patients who underwent transplantation within the last 1 year or for specific medical situations. In other cases, the attending physician continued telephonic visits for follow-up on the previously scheduled date. Since May 6, 2020, the emergency department of the institution was unified into a Care Unit of the Hospital Sao Paulo. Since May 15, 2020, patients with indication for hospitalization for other post-transplantation complications are subjected to RT-PCR collection. Among the 499 patients admitted for other complications, 7 (1.4\%) showed positive RT-PCR test results for SARS-CoV-2.
In these circumstances, a telemedicine department was created to educate, guide, and assist symptomatic or COVID19-positive patients; this was associated with active tracking, through telephone contact, for residents in the regions most affected by SARS-CoV-2 infection. The reference network of each patient was mapped, and clinical resolution was oriented in units closer to their personal residence, respecting the organization of the SUS line of care and using the reference and counter reference mechanisms. Diagnosed COVID-19 patients who remained isolated at home received telephone follow-up periodically, which was based on the severity of their case. Those admitted to other institutions were monitored through regular contact with the local assistant health care team.

Between March 3, 2020, the date on which the first patient with diagnosis of COVID-19 was registered in the institution, and August 28, 2020, there were 237 notifications of potential donors and 70 effectuations (30\%) in our Organ Procurement Organization and a total of 375 transplants with deceased donors, as a result of the additional receipt of organs from other organ procurement organizations of the state and the country (Figure 1). When compared with the same period in 2019 , there was a maintenance of the number of notifications and an increase of $19 \%$ in the number of transplants involving deceased donors. On the other hand, there was a fivefold reduction in the volume of interventional transplants (102 versus 22 procedures). The patient and graft survival within 30 days remained similar when compared with that of the previous year. Among the

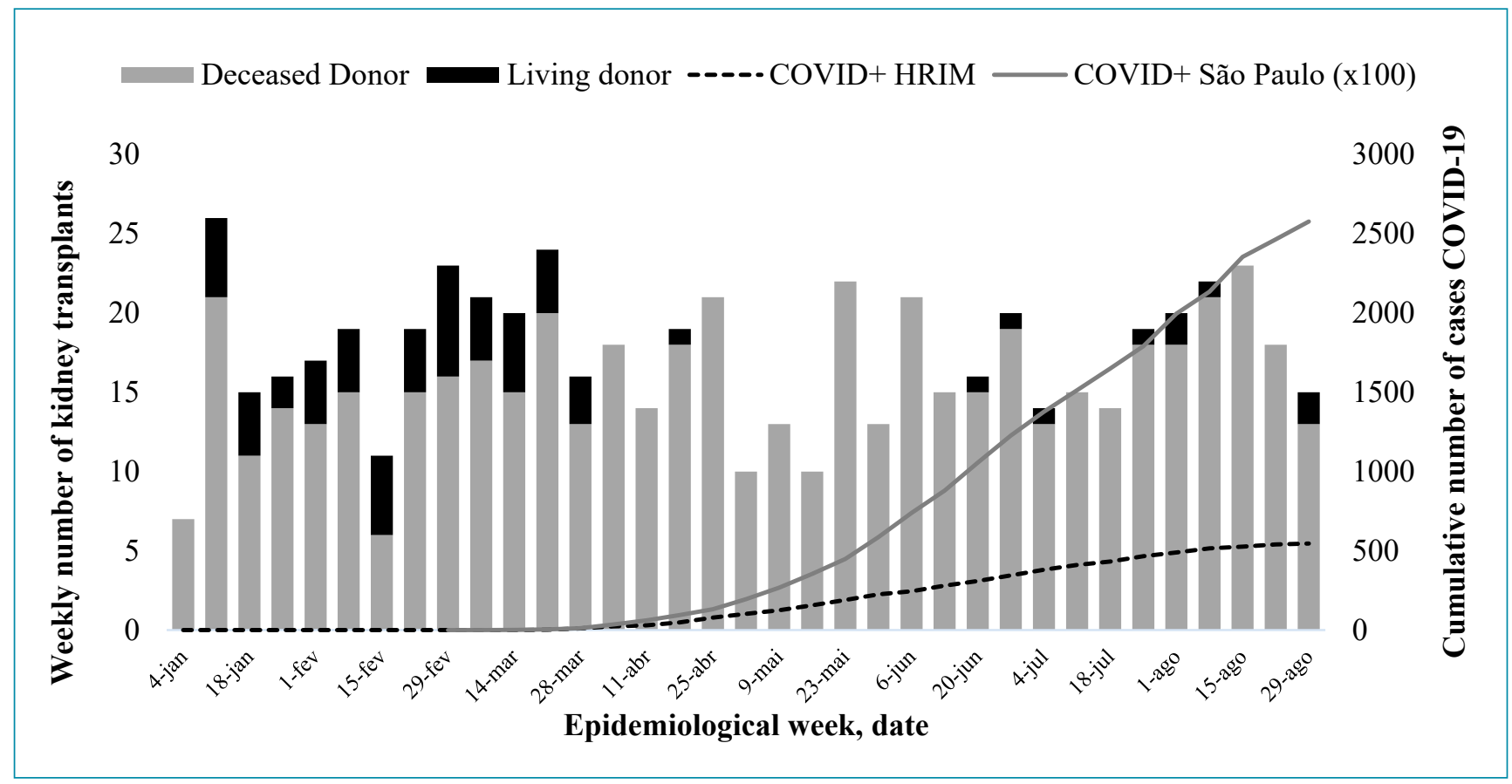

Figure 1. Number of new kidney transplants per epidemiological week during the COVID-19 pandemic among kidney transplant recipients of HRIM and the general population of São Paulo city (100x). 
transplanted patients during the pandemic period, 16 developed COVID-19 by acquiring the infection in the local community, 14 readmitted, and 2 died due to the disease.

Among the 11,875 patients transplanted in outpatient follow-up, there were 546 patients with a confirmed diagnosis of COVID-19. Out of these, 372 required hospitalization and 167 required mechanical ventilation. Regarding immunosuppression during hospitalization, there was transient interruption of an antimetabolite in $69(13 \%)$ patients, while interruption of all immunosuppressive drugs except steroids was necessary in $178(32 \%)$ patients. Acute renal graft dysfunction occurred in $286(52 \%)$ of the individuals and 167 (30\%) required hemodialysis. There were 148 deaths, resulting in an overall fatality rate of $27 \%$. The mortality rate was $39 \%$ among 372 patients who required hospitalization, $84 \%$ among those who required mechanical ventilation, and $85 \%$ when on mechanical ventilation and hemodialysis.

\section{DISCUSSION}

The strategies for confronting the pandemic were discussed and disseminated in all media, with massive participation of the transplant community. This communication network allowed the adoption of similar measures by several organ harvesting and transplant programs, with extensive discussions of alternatives based on the local epidemic of the disease. In the Brazilian Association for Organ Transplantation, we participated in many vital activities exchanging experience in facing the pandemic and creating awareness that the national transplantation program would be partially impacted. However, as the pandemic has a migratory character and the country has a continental dimension, we always understood that it would be possible to keep transplants concentrated in the less affected regions and we coined a phrase: "the soul of transplantation cannot die." Thus, the capillarity of the national transplant activity was kept active. The result was a national number of transplants slightly above $50 \%$ in relation to the same period last year, which guarantees all the social achievements of this activity, as well as the maintenance of civil society involvement in organ donation throughout the country. In the state of Saa Paulo, together with the State Transplant Center and the Regional Organ Procurement Organizations, the teams remained in the front line, making all their energy available, which resulted in the support of the programs with more than 30 years of history in the field of transplantation.

\section{CONCLUSIONS}

The COVID-19 pandemic, which is unprecedented in terms of geographic spread, poses a serious threat to overall public health and, specifically, to the solid organ transplant program. Thanks to the coordinated sequence of preventive actions and measures from a network of interprofessional and inter-institutional collaborations, it has been possible, so far, to guarantee safe access for patients with end-stage chronic kidney disease to kidney transplantation and its follow-up. In times of great challenges like COVID-19 pandemic, this initiative represents an example of relative success in maintaining the transplant program without compromising the safety of health professionals and transplanted patients who are in the follow-up.

\section{AUTHORS" CONTRIBUTION}

JMP: Conceptualization, Methodology, Data curation, Formal analysis, Writing - original draft. MPC: Conceptualization, Methodology, Data curation, Formal analysis, Writing - original draft. LAV: Conceptualization, Methodology, Data curation, Formal analysis, Writing - original draft. RAF: Methodology, Data curation, Formal analysis, Writing - original draft. MRN: Data curation, Supervision, Formal analysis, Writing - original draft. RDF: Conceptualization, Data curation, Formal analysis, Writing - original draft. SBSM: Data curation, Supervision, Writing - original draft. DWCLS: Validation, Writing - original draft. WFA: Writing - original draft. MLSV: Conceptualization, Writing - original draft.

\section{REFERENCES}

1. World Health Organization. WHO coronavirus disease (COVID19) dashboard. [Online] Geneva: World Health Organization; 2020. [cited on Jul. 09, 2020]. Available from: https://covid19. who.int/

2. Neves PDMM, Sesso RCC, Thomé FS, Lugon JR, Nascimento MM. Brazilian dialysis census: analysis of data from the 20092018 decade. J Bras Nefrol. 2020;42(2):191-200. https://doi. org/10.1590/2175-8239-JBN-2019-0234
3. Associação Brasileira de Transplante de Órgãos. Dimensionamento dos transplantes no Brasil e em cada Estado (2012-2019). RBT [Internet] 2019[cited on Jul. 09, 2020];XXV(4):3-6. Available from: http://muw. abto.org.br/abtov03/Upload/file/RBT/2019/RBT-2019-leitura.pdf

4. Pestana JM. A pioneering healthcare model applying large-scale production concepts: principles and performance after more than 11,000 transplants at Hospital do Rim. Rev Assoc Med Bras (1992). 2016;62(7):664-71. https://doi.org/10.1590/1806-9282.62.07.664 